\title{
La mosca de la fruta de la piña (Melanoloma viatrix Hendel) asociada a la mancha con galerías y experiencias de control en la selva central del Perú
}

\author{
Pineapple fruit fly (Melanoloma viatrix Hendel) associated with \\ gallery-type spot and control experiences in the central Peruvian jungle \\ Segundo Bello Amez ${ }^{1 *}$, Ricardo Borjas Ventura ${ }^{1}$, Leonel Alvarado Huamán ${ }^{1}$, \\ Noel Bello Medina ${ }^{1}$,Viviana Castro-Cepero ${ }^{1}$, Alberto Julca-Otiniano ${ }^{1}$
}

\begin{abstract}
RESUMEN
En el Perú la piña (Ananas comosus var. comosus) tiene 14000 ha cultivadas, con un rendimiento promedio de 22 t/ha. Los cultivares comerciales en orden de importancia son 'Samba', 'Hawaiana', 'Pucalpina', 'Roja Trujillana', 'Cayena Lisa' y 'Golden' (MD-2), que se siembran en las diferentes regiones del país, pero las mayores áreas se encuentran en la zona conocida como la selva central. La piña tiene diversos problemas fitosanitarios, pero el principal es la llamada "mancha del fruto" causada por Penicillium funiculosum y Fusarium moniliforme. Existen tres tipos de mancha: "mancha negra seca", "mancha negra húmeda" y "mancha con galerías". Esta última es la más importante y está asociada a la "mosca de la fruta de la piña" (Melanoloma viatrix). En este trabajo se presenta una revisión sobre la biología de la "mosca de la fruta de la piña", se describe la "mancha con galerías" y se exponen las experiencias desarrolladas para su control en el país.
\end{abstract}

Palabras clave: protección mecánica, manejo integrado, mosca de la fruta, mancha de la fruta.

\section{ABSTRACT}

In Peru, the pineapple (Ananas comosus var. comosus) has 14000 cultivated hectares, with an average yield of 22 t/ha. Commercial cultivars in order of importance are'Samba','Hawaiana','Pucalpina','Roja Trujillana','Cayena Lisa' and 'Golden'(MD-2) which are cultivated in different regions of the country, but the largest areas are in the central jungle. The pineapple has several phytosanitary problems, but the most important is the "fruit spot" caused by Penicillium funiculosum and Fusarium moniliforme. There are three types of spots: "dry black spot", "wet black spot" and "spot with galleries", the latter being the most important and associated with the "pineapple fly" (Melanoloma viatrix). This paper presents a review of the biology of the "pineapple fly", a description of the "spot with galleries" and the experiences developed for its control in the country.

Keywords: mechanical protection, integrated management, fruit fly, fruit spot.

\section{Introducción}

La piña [Ananas comosus var. comosus (L.) (Merr.), Coppens y Leal] (Coppens d’Eeckenbruge y Leal, 2003) es una planta herbácea perenne monocotiledónea que pertenece al orden Bromeliales y familia Bromeliaceae (Py et al., 1987). Crece en generaciones sucesivas y puede sobrevivir durante muchos años; es de porte variable y su sistema reproductivo predominantemente vegetativo. Presenta un tallo corto de consistencia herbácea de entrenudos muy próximos. El sistema radicular es pequeño, forma un conjunto denso, poco profundo y frágil. Las hojas son erectas, angostas y están adheridas al tallo formando una espiral compacta con un número variable que va desde 40 a 80 hojas por planta. Las hojas se clasifican en varios tipos, pero la hoja "D" es la de mayor importancia (Py et al., 1987) y es útil para estimar las necesidades nutricionales de la planta,

\footnotetext{
1 Grupo de Investigación: Agricultura y Desarrollo Sustentable en el Trópico Peruano. Facultad de Agronomía. Departamento de Fitotecnia. Universidad Nacional Agraria La Molina. Lima (Perú).

* Autor para correspondencia: sbello@frutasgolden.com
} 
así como su estado de crecimiento. El pedúnculo es una simple prolongación del tallo que soporta el fruto donde se desarrollan los bulbillos. La inflorescencia produce un número variable de flores de color violáceo y el fruto es múltiple. Al final del crecimiento la planta de piña presenta diferentes tipos de material vegetal como hijuelos de base de planta, de tallo, bulbillos y corona, que sirven de material de propagación para iniciar nuevas plantaciones.

La piña se cultiva en las regiones comprendidas entre los paralelos $30^{\circ}$ al norte y sur del Ecuador; sin embargo, el crecimiento y la producción son afectados cuanto más se aleja de la línea ecuatorial. Con respecto a la altitud, la piña crece bien de 0 hasta $1200 \mathrm{msnm}$. Altitudes superiores a las indicadas pueden causar problemas en el crecimiento y desarrollo del cultivo (Py et al., 1987). La temperatura para el crecimiento y desarrollo de la piña está entre 16 y $32{ }^{\circ} \mathrm{C}$; el crecimiento de la planta disminuye rápidamente con temperaturas medias por debajo de $15^{\circ} \mathrm{C}$ y superiores a $32{ }^{\circ} \mathrm{C}$. La precipitación para la producción óptima está entre 1000 y $1500 \mathrm{~mm} / \mathrm{año}$, con una buena distribución durante el año. En San Ramón/ Chanchamayo las lluvias anuales son de 1850 $\mathrm{mm}$, y se observa déficit de agua en los meses de junio, julio y agosto (Bello y Julca, 1994). La radiación influye en la formación de carbohidratos y consecuentemente en el desarrollo de la planta y su producción. Con poca luminosidad el fruto llega a la madurez opacado, mientras que con adecuada radiación presenta un aspecto brillante. La piña crece en una amplia gama de suelos desde orgánicos, volcánicos, arenosos, degradados, hasta secundarios y de pasturas. En general los suelos que presenten buen drenaje, buena aireación y que no tengan problemas de compactación son los más favorables (Py et al., 1987).

La piña hoy en día es un componente importante de la dieta alimenticia y se consume en todos los rincones del mundo porque es deliciosa, sana y nutritiva. En el ámbito internacional la demanda de la piña aumentó por el cambio de los hábitos alimenticios de los consumidores, debido al alto contenido de fibra, vitamina $\mathrm{C}$, su agradable sabor y aroma. El comercio mundial de la piña como fruta fresca se ha transformado en los últimos quince años como no ha sucedido con otras frutas. De acuerdo a la FAO, de 1995 a 2007, es decir, en solo doce años, el consumo de piña se incrementó en 359\%. Durante este proceso, Costa Rica se ha posicionado en el mercado mundial de fruta fresca con un $68 \%$ seguido de Filipinas con $12 \%$. En el Perú el cultivo de la piña se ha incrementado considerablemente en estos últimos años. Según el Ministerio de Agricultura, al año 2016 se tenía cerca de 14,000 hectáreas, con un rendimiento promedio de $22 \mathrm{t} / \mathrm{ha}$. Los cultivares comerciales en orden de importancia son 'Samba', 'Hawaiana', 'Pucalpina' y 'Roja Trujillana', 'Cayena Lisa' y 'Golden' (MD-2), que se siembran en las diferentes regiones del país, pero las mayores áreas se encuentran en la región Junín (Chanchamayo y Satipo), en la zona conocida como la selva central.

La piña, igual que otras especies cultivadas, tiene diversos problemas fitosanitarios y en el Perú el principal, sin lugar a dudas, es la llamada "mancha del fruto". Según Julca y Bello (2005), esta enfermedad es causada mayormente por Penicillium funiculosum y en menor grado por Fusarium moniliforme (Guérout, 1974; Rohrbach y Pfeiffer, 1976; Mourichon, 1991), y por diferencias en los síntomas se les clasifica como "mancha negra seca" (leathery pocket, MNS) y "mancha negra húmeda" (black spot, MNH). En nuestro país existe un tercer tipo denominado "mancha con galerías" (MCG), también causada por los mismos patógenos y es el más importante (Bello et al., 1997). La primera está asociada con la población de ácaros que viven en la inflorescencia de la piña y la segunda con el nivel de precipitación en la zona de producción (Py et al., 1987). El tercer tipo está asociado con la "mosca de la fruta de la piña" (Melanoloma canopilosum $=$ Melanoloma viatrix) (Bello et al., 1997) y puede dañar más de la mitad de la fruta cosechada. Actualmente se controla protegiendo la inflorescencia, pero algunos profesionales consideran que esta práctica ya no es necesaria y que basta con la aplicación de fungicidas e insecticidas, una decisión errónea si se considera que el área cultivada aumenta con las variedades introducidas, como 'Cayena Lisa' y 'Golden', además de la presencia del insecto en el área boscosa. Esto muestra un desconocimiento del problema y el nivel de daños que puede alcanzar si no se toman las medidas adecuadas de control. Por ello en este trabajo se presenta una revisión de la biología de la "mosca de la fruta de la piña" (M. viatrix), se describe la "mancha con galerías" y se exponen las experiencias desarrolladas en el Perú para su control. 


\section{La "mosca de la fruta de la piña" Melanoloma viatrix Hendel}

Taxonomía. La "mosca de la fruta de la piña" pertenece al orden Diptera, superfamilia Tephritoidea y familia Richardiidae. Está compuesta por 30 géneros y 175 especies, la mayoría de las cuales se alimentan de plantas o son saprófagos de material vegetal en descomposición (PérezGelabert y Thompson, 2006). Solo la especie Melanoloma loew ha sido reportada como plaga de la piña. En Perú la primera referencia fue realizada por Figueroa et al. (1970), quienes la registran atacando frutos maduros de piña en las zonas de Pichari (Cusco) y San Ramón (Junín) en 1967; y posteriormente por Bello et al. (1997), quienes la citan como Melanoloma canopilosum Hendel, 1933. El género Melanoloma sp. se distingue de otros de la familia Richardiidae por poseer una vena dm-cu ubicada en la mitad superior de la celda discal y por las genitalias de los machos y hembras (Hancock, 2010). En el 2004 fue identificado como Melanoloma viatrix Hendel 1911 por SENASA (Bello et al., 2008). Arellano et al. (2015), en consulta al Dr. Korytkowski, especialista en Tephritoidea, confirmaron $a M$. viatrix como la misma especie que se cita para otros países de la Región Neotropical (Arévalo y Osorio, 1995; Boscán et al., 2000; Montilla et al., 2008; Alvarado et al., 2010) (Figura 1).

Hábitos. Los insectos de la familia Richardiidae viven en las zonas de bosque tropical húmedo y neotrópico y sus hábitos son poco conocidos; sin embargo, se considera que son carpófagos secundarios y los mayores daños se encuentran en las plantaciones cercanas a las áreas boscosas. Arévalo y Osorio (1995) señalan que la piña afectada por esta plaga está establecida en laderas montañosas, con clima de bosque húmedo tropical y con precipitaciones cercanas a $5,000 \mathrm{~mm}$ anuales en Antioquia (Colombia). En el campo generalmente se les observa a tempranas horas de la mañana o en las tardes, desplazándose en los frutos con movimiento lateral agitando las alas

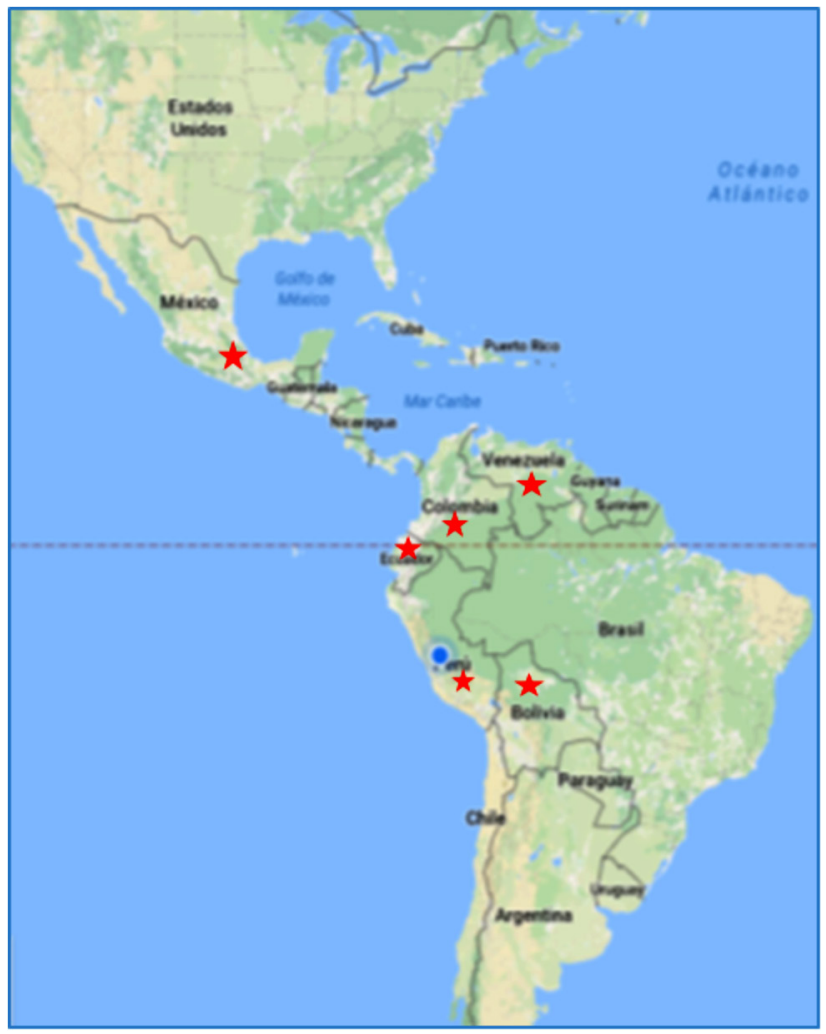

Figura 1. Distribución de la "mosca de la fruta de piña" Melanoloma viatrix Hendel en América (países señalados con una estrella roja). 
constantemente, buscando las hendiduras de los "ojos" o "frutillos" y generalmente ovipositan en masas, en la inserción de las brácteas florales de los frutos (Figura 2A).

Biología. Los huevos son de color blanco, de forma alargada y ahusada en los extremos y ovipositados en pequeños grupos o en masa (Arévalo, 1996; Morales y López, 2001a; Moreno y López, 2001b; Alvarado et al., 2010; Arellano et al., 2015), y eclosionan a los 3 a 5 días posteriores a la postura. Las larvas barrenan y penetran al fruto alimentándose de este. En algunos casos se han encontrado hasta 150 larvas por fruto (Alvarado et al., 2010). Las larvas son vermiformes, ápodas y de color blanco amarillento ahusadas. En el último estadio se puede observar la masa encefálica de un color más oscuro y esta característica se nota claramente en las Figuras 2B y C. Al terminar su desarrollo las larvas salen del fruto totalmente maduro y fermentado cayendo al suelo (casos extremos), donde empupan completando su ciclo biológico. La pupa es una cápsula de forma más o menos cilíndrica, de color marrón rojizo (Figura 2D), de una longitud de 4 a $5 \mathrm{~mm}$ (Bello et al., 1997).

Los adultos de la "mosca de la fruta de la piña" (Figura 2A) son de tamaño pequeño, de $12 \mathrm{~mm}$ de extensión alar y 5-6 mm de longitud de cuerpo, y de color negro oscuro. El tórax está cubierto con abundante micro pubescencia. Una característica resaltante son las alas, donde la nervadura coaxil presenta ligeras manchas de color negro que caracterizan a esta especie. El abdomen es de color negro. La hembra presenta un ovipositor poco conspicuo, pequeño, flácido y pubescente, por lo que necesitan ranuras, intersticios y/o hendiduras de las brácteas florales o el centro del "frutillo u ojo" para dejar sus posturas (Figura 2A). Los adultos tienen una característica resaltante en las alas que permite reconocerlos fácilmente y es una franja oscura en el borde de la vena costal, la cual se extiende hasta un poco después de la denominada vena media. Asimismo, tienen una mancha oscura a la altura de la vena radial media. $\mathrm{El}$ adulto mueve permanentemente sus alas, con desplazamientos horizontales dirigidos hacia la cabeza. Las hembras se vuelven más activas en busca de los lugares de oviposición.

El ciclo de vida de $M$. viatrix en condiciones de gabinete (San Ramón, Chanchamayo) es de 41 a 47 días, distribuidos de la siguiente manera: de oviposición a eclosión tiene una duración de 4 a 5 días, el estadio larvario de 14 a 16 días, la prepupa 2 días, en estado de pupa permanece de 14 a 16 días y finalmente el paso al estado adulto tiene una duración de 6 a 8 días (Bello, 2009; observación personal). Resultados similares han sido reportados en otros países productores de piña como Colombia y Venezuela (Morales y López, 2001; Alvarado et al., 2010).

Distribución. La familia Richardiidae es pequeña y está restringida a las regiones neotropical y tropical de América (Pérez-Gelabert y Thompson, 2006). La "mosca de la fruta de la piña" en el Perú se ha registrado en la mayoría de las zonas productoras a excepción de la costa norte del país. Además del Perú, se tienen reportes de daños por este insecto en Colombia y Venezuela, donde está causando serios problemas en el cultivo (Arévalo y Osorio, 1995; Boscán et al., 2000; Montilla et al., 2008; Alvarado et al., 2010). También existen reportes de la presencia de este insecto en
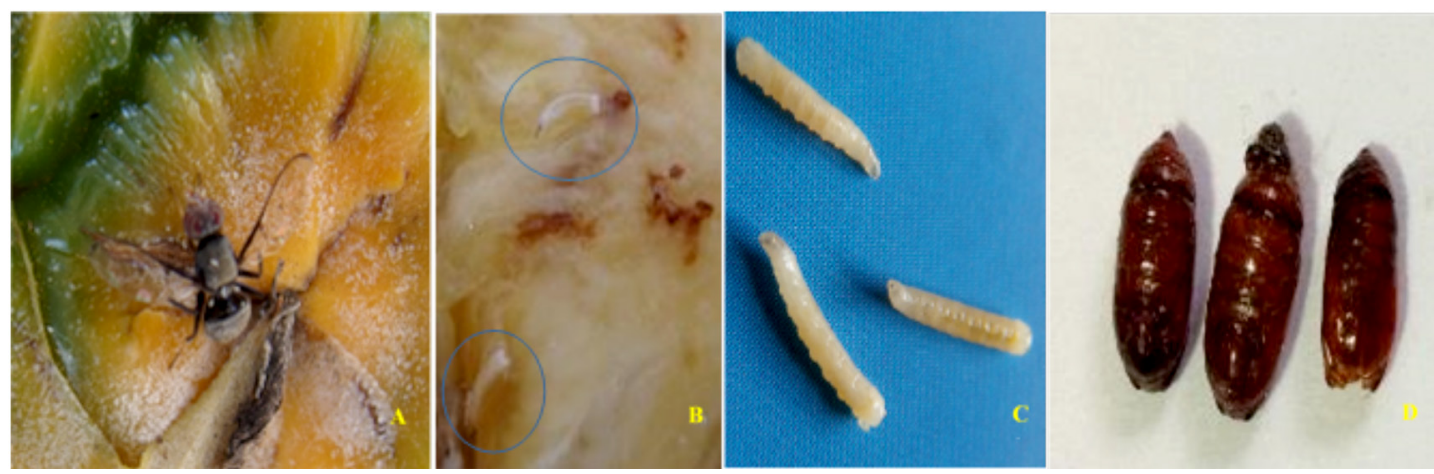

Figura 2. "Mosca de la fruta de la piña" M. viatrix, A: adulto ovipositando en los "ojos" de fruta madura, B y C: larvas en la pulpa de la piña y larvas extraídas, D: Pupas (Fotos: Segundo Bello). 
Bolivia (Skeyskal, 1968), Ecuador (Rogg, 2000) y en México, como se muestra en la Figura 1. No se han registrado hospederos alternos. Se conoce que viven en el bosque húmedo sobre la materia orgánica en descomposición. Las larvas de las moscas de la familia Richardiidae son saprófagos (Hancock, 2010) y viven generalmente en hojarasca, en malezas, en excrementos frescos y en descomposición.

\section{La "mosca de la fruta de la piña" $y$ su relación con la "mancha con galerías"}

Como se ha señalado anteriormente, en Perú se han reportado tres tipos de manchas del fruto de la piña (Figura 3), pero la más importante es la "mancha con galerías" que está altamente correlacionada con la población de $M$. viatrix con un $\mathrm{r}=0,6656^{* *}$ (Bello y Julca, 2005). En la selva central, los daños ocasionados por las manchas pueden llegar hasta el $80 \%$ de los frutos, cuando la cosecha se produce en la época seca, es decir, en los meses de junio, julio y agosto (Bello, datos no publicados). Inicialmente, la presencia de $M$. viatrix en los campos de piña no recibió la menor atención y no se relacionó con la "mancha con galerías", pues se consideraba que el problema se circunscribía a frutas maduras abandonadas en el campo, y que no era algo serio para el cultivo. Sin embargo, con la introducción de la 'Cayena Lisa' por los años 80 y los estudios realizados entre 1983 y 1987, se confirmó la importancia económica de esta especie (Arellano, 2015). Recién en la década del 90 se determinó la relación entre la mosca y la "mancha con galerías", problema que limitaba el establecimiento del cultivar 'Cayena Lisa' en la selva central de nuestro país (Bello et al., 1997; Bello y Julca, 2005; Arellano et al., 2015). La "mosca de la fruta de piña" ataca todos los cultivares de piña en el Perú. Por ejemplo, en la localidad de Toterani (Chanchamayo) se registró hasta el $80 \%$ de frutos dañados en el cultivar local 'Samba' (Arellano, 2015). Años más tarde, Bello et al. (1997) reportaron frutos con "mancha con galerías" con una incidencia entre 20 y $70 \%$ en los cultivares 'Samba' y 'Cayena Lisa'. En Colombia, Arévalo (1996) registró daños por esta plaga de hasta un $100 \%$ en 'Cayena Lisa' y en menor proporción en la variedad local 'Manzana'. En Venezuela, Alvarado et al. (2010) reportaron daños de hasta un $70 \%$ en las variedades locales 'Valera Amarilla' y 'Valera Roja'.

Síntomas y daños. Los síntomas y daños de la "mosca de la fruta de la piña" pueden ser externos e internos. Ello dependerá de los cultivares, la presencia o no de los hongos responsables de la "mancha del fruto" y el momento de ataque al fruto. En cultivares de fruto con cáscara de color oscuro no se observan síntomas externos y solo se sabe que están dañados al cortarlos y ver la pulpa. Esto sucede en la 'Samba', 'Roja Trujillana' y 'Pucalpina'. En cambio, en frutos con cáscara de color claro (amarillo, verde amarillento) como la 'Cayena Lisa' y 'Golden' y con un grado de madurez 3, 4 y 5, se observan síntomas externos de maduración irregular que se ha denominado "maduración tipo mosaico", porque el fruto, externamente, presenta áreas verdes no maduras y áreas maduras de color amarillo (Bello, et al., 1997). Las áreas verdes corresponden a zonas de oviposición e inicio del daño de las larvas y las áreas de color amarillo son zonas sanas, de maduración
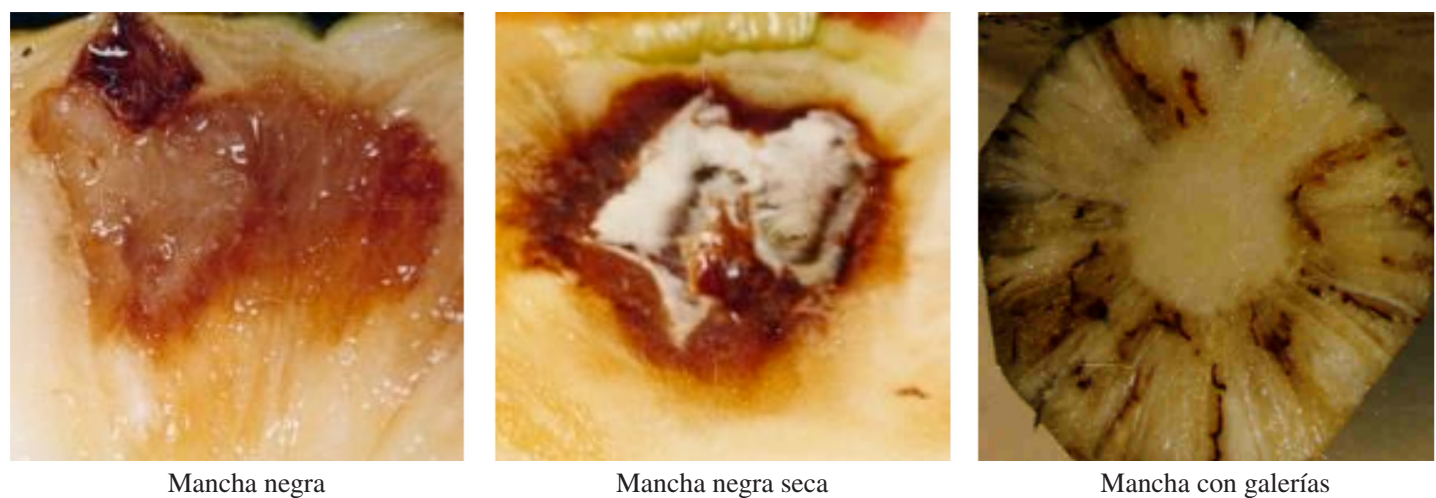

Mancha con galerías

Figura 3. Los diferentes tipos de manchas del fruto en la piña (Tomado de Julca y Bello, 2005). 


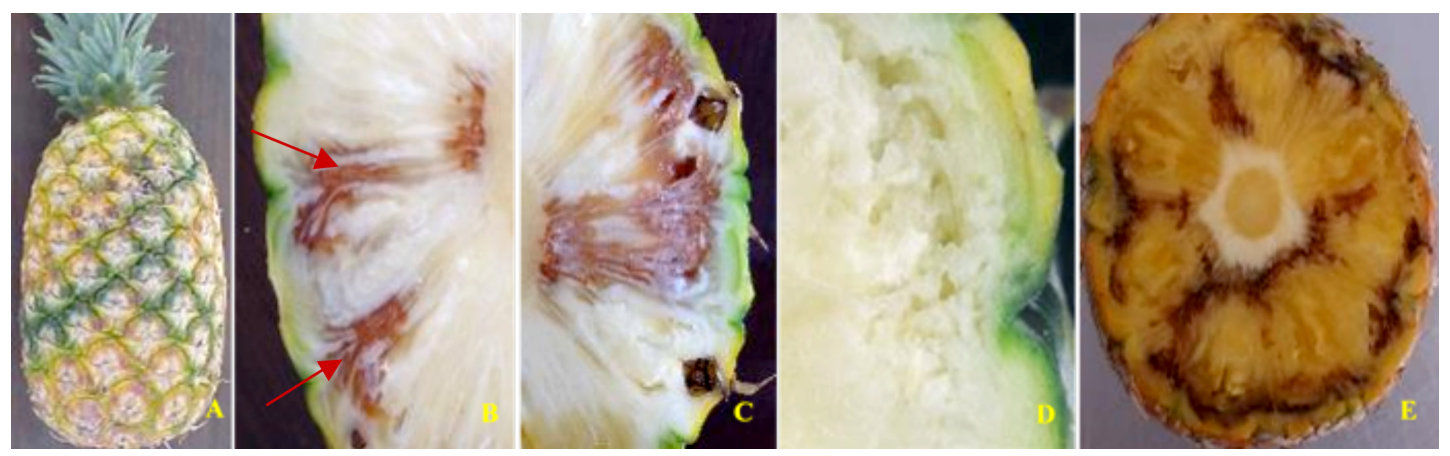

Figura 4. Síntomas y daños de M. viatrix en piña ‘Golden’ A: síntoma externo tipo mosaico en fruta sobre madura, B: la flecha indica el lugar de oviposición en la unión de los “ojos", C: daño llega hasta el "corazón” de la pulpa de fruta D: síntoma de barrenamiento de pulpa sin mancha (daño tardío) cercano a la cosecha y E: síntoma típico MCG (Fotos: Segundo Bello).

normal (Figura 4A). La maduración irregular del fruto también ha sido reportada en Venezuela en cultivares de piña locales (Alvarado et al., 2010).

Para entender cómo se producen los síntomas internos en el fruto, es importante recordar que el tiempo que transcurre entre el Tratamiento de Inducción Floral (TIF) y la cosecha varía de 160 a 180 días, según el tamaño del fruto. Los síntomas y daños cambian según el momento en que la mosca realizó la oviposición en el fruto, y por ello podrían tener las siguientes características:

Si la "mosca" oviposita en frutos en desarrollo (125-130 días después del TIF), las larvas de los primeros estadíos migran dentro de la pulpa, principalmente por la zona del exocarpo de los frutillos, produciendo micro galerías que se necrosan por la presencia del complejo fungoso. Luego se juntan y forman lo que se denomina "mancha con galerías" MGC (Figura 4E).
Si la oviposición se produce en frutos de mayor desarrollo (135 días después de la TIF), las larvas se alimentan de la pulpa, barrenan el fruto y llegan hasta el corazón de la fruta (Figura 4B, 5C). Las manchas con galerías se juntan con los otros tipos de manchas, formando un complejo de manchas, sin patrón definido y terminan dañando totalmente la fruta.

Cuando el insecto oviposita cerca de la cosecha (fruta pintona), las larvas no favorecen la presencia del complejo fungoso y básicamente se alimenta en la zona cercana a la cáscara del fruto, como se observa en la Figura 4D. La pulpa afectada no presenta ningún tipo de mancha y las larvas son imperceptibles para el consumidor común.

\section{Manejo de la "mosca de la fruta de la piña" asociada a la MCG}

El manejo integrado de plagas (MIP) ha sido definido como "el sistema de manejo, que utiliza
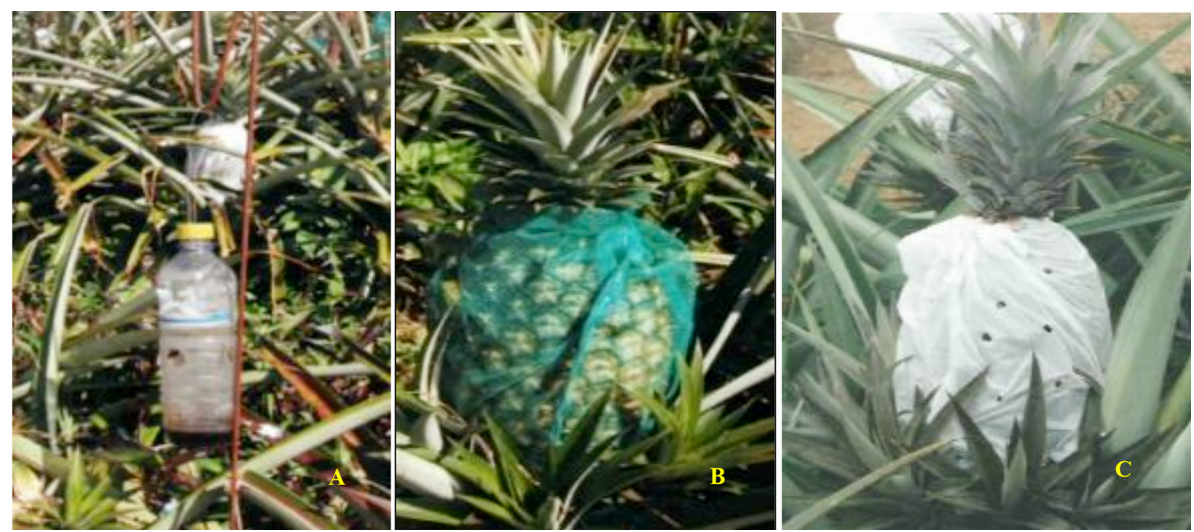

Figura 5. Métodos de control de M. viatrix; A: trampa casera, B: malla de PVC y C: manga de polietileno (Fotos: Segundo Bello). 
todas las técnicas y métodos adecuados de forma compatible con el medio ambiente, manteniendo las poblaciones nocivas a niveles por debajo del umbral económico" (Romero, 2004). Puede realizarse a tres niveles, donde el objeto de control (a) es una sola especie de plaga, (b) un rango de plagas y (c) un complejo de todos estos organismos nocivos para el cultivo. Sin embargo, para el desarrollo del MIP se requieren conocimientos básicos con los que se logra una comprensión más real y precisa de la influencia de los factores bióticos y abióticos que regulan el comportamiento de las plagas. Los conocimientos básicos para el MIP son la identificación de las plagas, el nivel de infestación, biología y ecología de las plagas, los umbrales de daño económico y métodos de control técnicamente efectivos, económicamente viables y seguros para el ambiente (Moreno y López, 2001a y b; Julca et al. 2008; Moreno y López, 2001a). Por ello, entre los años 1990 y 1995 se realizaron un conjunto de ensayos para diseñar un programa de manejo integrado como a continuación se describe.

Control Genético. En el Perú se ha observado que todos los cultivares comerciales son susceptibles a la "mancha del fruto". Pero cuando se evaluaron las diferentes accesiones del banco de germoplasma de piña en Chanchamayo, se encontró que el genotipo 89-06 (actualmente conocida como Hawaiana), tuvo solo un $6 \%$ de frutos manchados, una incidencia menor a la de otros genotipos que mostraron frutos con daños superiores al 20\% (Bello et al., 2015). Esta es una de las razones por las cuales las colecciones de germoplasma de piña son de vital importancia en la determinación de resistencia, tolerancia o susceptibilidad a las diferentes plagas y enfermedades (Duval et al., 1995; Leal y Coppens d'Eeckenbrugge, 1996; Cabral y Coppens d'Eeckenbrugge, 2002).

Control Químico. En un ensayo de campo para determinar el efecto de diferentes fungicidas químicos sobre la incidencia de la "mancha del fruto" en piña 'Cayena Lisa', se encontró que los fungicidas Benomyl, Tiabendazol y Tebuconazol controlaron significativamente la "mancha del fruto". Los resultados fueron bastante alentadores, pero se señaló que además de la aplicación de fungicidas, serán necesarias medidas complementarias que, por un efecto aditivo, permitan disminuir aún más la incidencia de la enfermedad, hasta llevarla a niveles económicamente no importantes. Se estima que en una plantación comercial de piña
'Cayena Lisa' (61 500 pl/ha) y usando el fungicida más eficaz de ese ensayo (Benomyl), se tendrían aún 8.370 frutos manchados que representarían pérdidas mayores a las $8 \mathrm{t} / \mathrm{ha}$. Por ello, se recomendó realizar otros estudios sobre el tema, por ejemplo, evaluar mezclas de fungicidas con insecticidas y/o acaricidas, por la asociación que existe entre la MNS y la población de ácaros en la inflorescencia y entre la MCG y la "mosca de la fruta de la piña" (Julca y Bello, 2005). En Colombia, Moreno y García (1996) reportaron que el uso de cebos tóxicos fue eficaz en el control de M. viatrix, con aplicaciones semanales y continuas de una mezcla de melaza, urea y Malathion 2.5 cada uno al 10\%o de producto comercial.

Control Etológico. Las trampas y los atrayentes son las herramientas básicas del monitoreo de las poblaciones y la base para la planificación de las medidas de control. Existen trampas artesanales y atrayentes elaborados a base de jugos de frutas, urea, melaza, orina y gallinaza, entre otros.

Se usan para capturar o destruir insectos, para detectar su presencia o determinar su ocurrencia estacional y abundancia con la finalidad de orientar su control. Las trampas comúnmente utilizadas para la detección de las "moscas de la fruta" de la familia Tephritide son la McPhail, Steiner y Nadel. La experiencia peruana muestra que el uso de trampas caseras no tuvo efecto en el control de la "mosca de la fruta de la piña" tal como se observa en la Figura 5A y en la Tabla 2.

Control Cultural. Es la utilización de las prácticas agrícolas ordinarias, o algunas modificaciones de ellas, con el propósito de prevenir los ataques de los insectos y de las enfermedades, proporcionando un ambiente lo menos favorable para su desarrollo, y disminuir sus daños. Con ese objetivo, una labor importante es el TIF, ya que permite concentrar la cosecha y por lo tanto hace posible el cuidado de la inflorescencia y posteriormente el fruto. También es importante, al final de la cosecha, recoger los frutos remanentes y enterrarlos en hoyos de un metro de profundidad que se tapan con tierra para evitar que los insectos emerjan a la superficie. Además es recomendable mantener el campo libre de malezas durante el desarrollo del fruto y de excrementos de animales y humanos, donde la mosca se reproduce fácilmente. En lo posible deben evitarse fuentes orgánicas cercanas a las plantaciones porque es el hábitat natural de la mosca. 


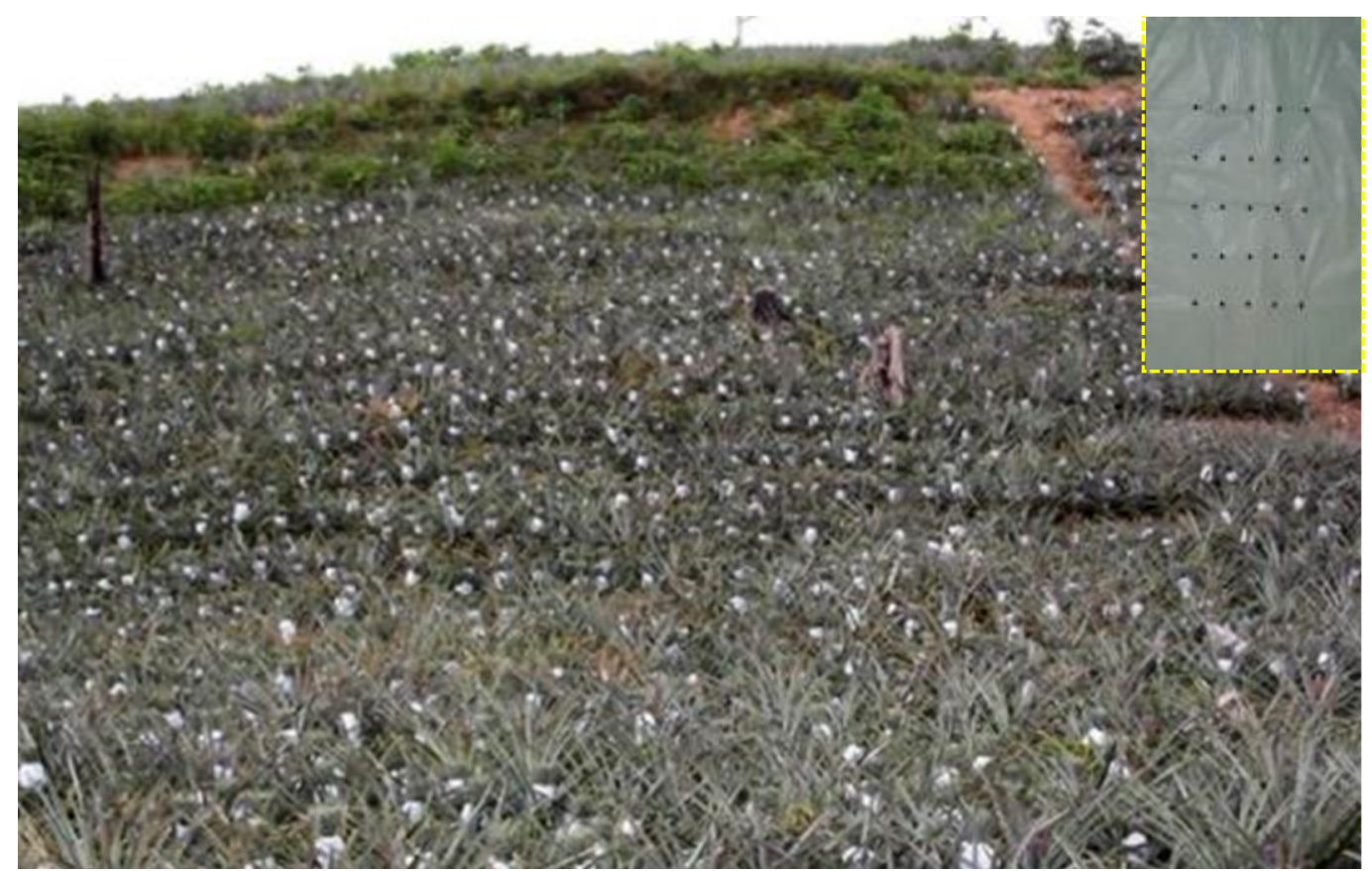

Figura 6. Campo de piña 'Cayena Lisa' con inflorescencias protegidas con "bolsa" de polietileno, la cual es abierta por los dos extremos, tiene 25 agujeros y mide 30 x $45 \mathrm{~cm}$ (Foto: Alberto Julca).

Control Mecánico. Este método consiste en la remoción y destrucción de los insectos y órganos infestados de las plantas, en este caso los frutos. También se refiere al uso de barreras físicas o de otros tipos, para evitar el contacto del insecto con el órgano que se protegerá. Para este caso, se hicieron ensayos protegiendo las inflorescencias de la piña con mallas PVC que aportaron información relevante para el control de la "mosca de la fruta de piña" asociada a la MCG. En la Tabla 1 se muestra que los frutos protegidos a los 75 y 105 días después de TIF no presentaron mancha; en

Tabla 1. Efecto del momento de la protección mecánica de la inflorescencia sobre la incidencia (\%) de los diferentes tipos de "mancha de la fruta" en piña 'Cayena Lisa' en Chanchamayo (Adaptado de Bello y Julca, 2005).

\begin{tabular}{crcr}
\hline \multirow{2}{*}{$\begin{array}{c}\text { Momentos de protección } \\
\text { (días después del TIF) }\end{array}$} & \multicolumn{3}{c}{$\begin{array}{c}\text { Frutos con diferentes } \\
\text { tipos de mancha }(\%)\end{array}$} \\
\cline { 2 - 4 } & MCG & MN & MNS \\
\hline 75 & 0,0 & 0,0 & 3,0 \\
105 & 0,0 & 0,0 & 4,3 \\
135 & 19,0 & 0,5 & 5,0 \\
165 & 72,5 & 1,5 & 5,3 \\
Testigo sin protección & 95,3 & 1,8 & 7,5 \\
\hline
\end{tabular}

cambio, los frutos protegidos a los 135,165 y el tratamiento testigo (sin protección) mostraron daños de $19 \%, 72,5 \%$ y 95,3\% respectivamente (Bello et al., 1997). Asimismo, Bello et al. (2008) determinaron que la protección de la fruta con mangas de polietileno con agujeros (Figura 5C) y con mallas PVC (Figura 5B) permitió un control total de la MCG asociada a M. viatrix (Bello y Julca, 2005; Bello et al., 2008), comparado con el uso de trampas caseras (Figura 5A) y el testigo sin protección. En estos tratamientos se registraron altos porcentajes de frutos dañados, así como el mayor número promedio de larvas/fruto (Bello et al., 2008), como se muestra en la Tabla 2.

Actualmente en el Perú esta técnica se usa en todas las zonas productoras de piña cultivada de forma tecnificada (Figura 6) y que mayormente trabajan con el cultivar 'Golden' (MD2). La protección mecánica se inicia a partir de los 90 días después del TIF y puede hacerse hasta 30 días después. Así el control de la "mancha del fruto" es del 100\%. En esta etapa la protección es sumamente fácil, pues la corona de la fruta en crecimiento permite sujetar alrededor de ella la manga ("bolsa") de polietileno. Una persona bien entrenada puede proteger entre 2500 y 3000 frutos/día, labor que es realizada generalmente por 
Tabla 2. Efecto de diferentes materiales en la protección mecánica de la inflorescencia y trampas caseras sobre la incidencia (\%) de los diferentes tipos de "mancha de la fruta" en piña 'Cayena Lisa' en Chanchamayo (Adaptado de Bello y Julca, 2008).

\begin{tabular}{|c|c|c|c|c|c|}
\hline \multirow[t]{2}{*}{ Tratamientos: } & \multirow{2}{*}{$\begin{array}{l}\text { Total de frutos } \\
\text { manchados }(\%)\end{array}$} & \multicolumn{3}{|c|}{$\begin{array}{c}\text { Frutos con diferentes tipos } \\
\text { de mancha }(\%)\end{array}$} & \multirow[t]{2}{*}{$\mathrm{N}^{\mathrm{o}}$ larvas/fruto } \\
\hline & & MNS & $\mathrm{MN}$ & MCG & \\
\hline Protección con malla PVC & 0,0 & 0,0 & 0,0 & 0,0 & 0,0 \\
\hline Protección con polietileno & 0,0 & 0,0 & 0,0 & 0,0 & 0,0 \\
\hline Trampas caseras & 63,0 & 0,7 & 1,1 & 61,2 & 1,8 \\
\hline Testigo sin protección & 70,0 & 0,4 & 1,2 & 68,4 & 2,3 \\
\hline
\end{tabular}

las mujeres, quienes tienen mejor predisposición para esta actividad. A pesar del éxito de este método de control, aún existen muchos agricultores que siguen aplicando pesticidas sin respetar el periodo de carencia y hasta unos cuantos días antes de la cosecha de la fruta, poniendo en alto riesgo la salud de los trabajadores, consumidores y el medio ambiente.

Pocas veces la aplicación de un solo método de control ha tenido un éxito duradero, y por ello la necesidad de desarrollar nuevos métodos de control o mejorar los ya existentes. Por ejemplo, una crítica que se hace a la protección mecánica de inflorescencias es que las "bolsas" de polietileno tardan mucho en degradarse y por lo tanto impactan negativamente en el medio ambiente. En el futuro sería conveniente usar "bolsas" de materiales más amigables con el medio ambiente que recientemente han aparecido en el mercado peruano. Bello y Julca (2005) ya han señalado la necesidad de explorar otros métodos de control con el objeto de desarrollar programas de manejo integrado. En ese sentido, las experiencias de otros países productores de piña son interesantes. Por ejemplo, en Venezuela, para el control biológico se ha reportado la presencia de un potencial parasitoide de pupas identificado como Spalangia drosophilae Ashmead (Hymenoptera: Pteromalidae), aunque tiene un bajo porcentaje de parasitismo (Montilla et al., 2007). En el mismo país, y como medida de control legal, se han declarado en cuarentena las zonas de producción infestadas de "gusano de la piña" (M. viatrix) y se ha restringido el traslado de todo material vegetal vivo a otras zonas del país, donde aún no se ha reportado la plaga. En Colombia, el manejo integrado de la "mosca de la fruta de la piña" ha sido aplicado exitosamente (Morales y López, 2001; Moreno y López, 2001a y b).

\section{Reconocimiento}

Los autores expresan su reconocimiento al Dr. Klaus Raven Buller (UNALM+), Dr. Hugo Villachica León (Grupo Frutas Golden) e Ing. Lambert Pie (INDALSA), así como a los especialistas del CIRAD/ FHLOR (Francia), Pierre Martin-Prevel, Alain Pinon, J. J. Lacoueilhe, J. Mourichon y J. L. Sarah, quienes, con sus ideas, visión y trabajo, ayudaron al desarrollo del cultivo de la piña en la selva central del Perú.

\section{Literatura citada}

Alvarado, C.; Balza, R.; Lacruz, L.; Durán, D. 2010. El gusano de la piña. Aspectos generales de biología y manejo. INIA-Divulga. 15: 13-16.

Arellano, G.; Vergara C.; Bello S.

2015. Plagas entomológicas y otros artrópodos en el cultivo de la piña (Ananas comosus var. comosus (L.) Merr., Coppens \& Leal) en Chanchamayo y Satipo, departamento de Junín, Perú. Ecología Aplicada, 14(2): 175-189.

Arévalo, P.E.

1996. Biología y hábitos del gusano de la piña Melanolma viatrix Hendel. Control de enfermedades y plagas en la piña. ICA, Colombia. Boletín de Sanidad Vegetal, 09: 41-43.

Arévalo, P.E.; Osorio M.

1995. La mosca de la piña, nueva plaga en cultivos del departamento de Antioquia. Control de enfermedades y plagas en la piña. ICA, Colombia. Boletín de Sanidad Vegetal, 09: 63-71.

Bello S.; Julca A.

2005. Mechanical protection of 'Smooth Cayenne' pineapple (Ananas comosus L. Merr.) inflorescence and fruit, and its effect on 'fruit rot' in Chanchamayo - Perú. Acta Horticulturae, 666: 241-245.

Bello S.; Julca, A.; Echevarría, I.C.; Cruz, R.

2015. Caracterización de genotipos de piña (Ananas comosus var. comosus) cultivados en la Amazonía del Perú. J. Interamer. Soc. Trop. Hort., 56: 13-19.

Bello, S.; Julca, A.; Villachica, H.

1997. Mancha de la fruta de piña tipo galerías asociada a Melanoloma canopilosum Hendel. Acta Horticulturae, 425: 493 -500. 
Bello, S.; Villachica, J.H.; Villachica, J.H. Jr.; Julca A. 2008. Efecto de la protección mecánica de la inflorescencia y el uso de trampas caseras en el control de la mancha de la fruta de la piña en la selva central del Perú. Proc. Interamer. Soc. Tropical Horticulture, 52: 88-92.

Boscán, N.; Rosales, C.; Godoy, F. 2000. La mosca del fruto de la piña Melanoloma viatrix (Diptera: Richardiidae). Agronomía tropical, 50(1): 135-140.

Cabral, J.R.S.; Coppens d'Eeckenbrugge, G. 2002. Abacaxizeiro. In C.H. Bruckner (ed.). Melhoramento de fruteiras tropicais. UFV, Viscosa, Brasil. pp. 37-61.

Duval, M.F.; Bernasconi, B.; Coppens d'Eeckenbruge, G. 1995. Manejo y evaluación de los recursos genéticos de piña en Martinica. Rev. Fac. Agron., (Maracay) 21: 147-155.

Figueroa, R.; Wolfe, H.; Franciosi, R.; Van Oordt, E. 1970. El cultivo de la piña en el Perú. Boletín Técnico No 75 Ministerio de Agricultura, Lima, Perú.

Guérout, R.

1974. Les taches noires de l'ananas. Fruits, 29(7-8): 489-499.

Hancock, E.G.

2010. Richardiidae (Richardiide Flies). En: Brown, B.V. Manual of Central American-Diptera: volume 2. Ed. 2010. NRC Research Press. Otawa. Canadá. pp. 871-880.

Julca, A.; Bello S.

2005. Control químico de la "mancha del fruto" en piña (Ananas comosus L. Merr.) cv. Cayena Lisa en Chanchamayo (Perú). Idesia (Chile), 23(2): 33-38.

Julca, A.; Julca, N.; Blas, R.; Bello, S.; Carhuallanqui, R.; Crespo, R. 2008. Experiencias para el manejo integrado de Cercospora nicotianae Ellis \& Everh en tabaco negro, localidad de Juan Guerra. Tarapoto. Perú. Idesia (Chile), 26(1): 15-27.

Leal, F.; Coppens d'Eeckenbrugge, G.

1996. Pineapple. In: Janick, J.; Moore, J.N. (eds.): Fruit Breeding. I. Tree and tropical fruits. Wileys and Sons. New York, US. pp. 515-557

Montilla, R.; García, J.; Lacruz, L.; Durán, D.

2007. Sphalangia drosophilae Ashmead (Hymenóptera Pteromalidae) parasitoide de pupas de la mosca de la piña Melanoloma viatrix Hendel (Díptera - Richardiidae) en Trujillo, Venezuela. Agronomía Tropical, 57(2): 107-112.

Montilla, R.; Lacruz, L.; Durán, D.

2008. Distribución Geográfica de Melanoloma viatrix Hendel (Diptera: Richardiidae) en Trujillo, Venezuela. Agronomía Tropical, 58(4). 403-407.
Morales, J.G.; López, J.

2001. Manejo de la mosca de la piña en Santander (Melanoloma viatrix Hendel). CORPOICA Regional 7-Bucaramanga/ Colombia. 24 p.

Moreno, J.; López J.

2001a. Embolsado del fruto, una técnica sostenible y competitiva para prevenir el daño de la mosca de la fruta de la piña (Melanoloma viatrix Hendel). CORPOICA Regional 7 - Bucaramanga- Colombia. 17 p.

Moreno, J.; López, J.

2001b. Estudios básicos para el manejo y control integrado de la mosca de la fruta de la piña (Melanoloma viatrix Hendel). Boletín de Investigación $\mathrm{N}^{\circ} 2$. CORPOICA Regional 7 - Colombia. 40 p.

Moreno, R.P.; García, A.

1996. Evaluación de cebos tóxicos en el manejo y control de Melanoloma viatrix. Sistemas de cultivo de la piña. ICA. Colombia. pp. 77-81.

Mourichon, X.

1991. Etude sur les maladies du fruit: Les taches noires et leathery pocket. Fruits, 46 (numéro spécial Ananas): 390-394.

Pérez-Gelabert, D.; Thompson, C.

2006. A new genus and species of Richardiidae (Diptera) from Hispaniola. Zootaxa, 1259: 21-32.

Py, C.; Lacoeuilhe, J.J.; Teisson, C.

1987. The pineapple: cultivation and uses. Ed. G.P. Maisonneuve et Larose, París, Francia. 452 p.

Rogg, H.

2000. Manejo integrado de plagas en cultivos de la Amazonía ecuatoriana. Escuela Superior Politécnica Ecológica Amazónica. IICA. Quito, Ecuador.

Rohrbach, K.G.; Pfeiffer, J.B.

1976. Field induction and etiology of pineapple interfruitlet corking, leathery pocket and fruitlet core rot with Penicilliumfuniculosum. Phytopathology, 66(2): 392-395.

Romero, F.

2004. Manejo integrado de plagas: las bases, los conceptos su mercantilización. Universidad Autónoma de Chapingo, Instituto de Fitosanidad. México. 103 p.

Steyskal, G.C.

1968. Family Richardiidae. A Catalogue of the Diptera of the Americas South of the United States. Part 53. Departamento de Zoologia, Secretaria da Agricultura. São Paulo, Brazil. 